\title{
AN ADAPTIVE ERROR-CHECK SOLUTION
}

Ying $\operatorname{Lin}^{1,2}$, Liang $\mathrm{Ge}^{2}$, Yueheng Sun ${ }^{1, *}$

ISchool of Computer Science and Technology, Tianjin University, Tianjin, China, 300072;

${ }^{2}$ College of management, Chongqing Jiao Tong University, Chongqing, China,400074;

* Corresponding author, Address: School of Computer Science and Technology, Tianjin University, Tianjin, China, 300072,Tel: +86-22-27401016,Email: yhs@tju.edu.cn

Abstract: Because of the traditional methods of video error checking are unfitted to the network environment in which the packet loss rate frequently changes, this paper proposes an adaptive error checking solution on the basis of estimation of the change trend of packet-loss rate. By setting the threshold value of packet-loss rate, the solution takes advantage of improved auto repeat request supported by RDP to realize real-time transportation in a low packet-loss rate, and uses improved forward error correction, a method of dynamically decollating images at the basis of varying sending rate, to solve the problem of error checking in a low transmitting rate.

Keywords: auto repeat request, forward error correction, packet-loss rate, transmitting rate

\section{INTRODUCTION}

Unstable Network seriously influences the real-time transportation of Video streams. When there exist great jams in the communicating channel, the quality of transportation decreases fiercely. If the rate of packet loss is more than 15 percents, streams are too broken to be rectified totally (Zhang et al., 2001). So in order to keep video complete and clear, the receiver always uses many methods checking errors to amend and restore video damaged partly. ARQ and FEC, which are used usually, can make a perfect effort in large scopes. However, as they are insensitive to the frequently changing situation of transporting channel, so they perform terribly in such a condition and worse bandwidth obviously (Shan et al., 2003). In a word, in the circumstance of dynamically varying bandwidth, it doesn't exist a ideal

Please use the following format when citing this chapter:

Lin, Y., Ge, L. and Sun, Y., 2009, in IFIP International Federation for Information Processing, Volume 294, Computer and Computing Technologies in Agriculture II, Volume 2, eds. D. Li, Z. Chunjiang, (Boston: Springer), pp. 1125-1134. 
solution to the real-time transportation of video streams with high quality (Fan et al., 2007). Considering, this paper proposals an adaptive method to solve the problem of checking error for streams in the condition of the rate of packet-loss infirmly varying.

\section{THE COMPARISON OF CURRENT ERROR- CHECK METHODS}

\subsection{Auto Repeat Request}

The principle of Auto Repeat Request (ARQ) method is that after checking error, the receiver sends the sender data message about the list of false data if there are some mistakes producing in the transportation (Zhang et al., 2004). Usually, the third method is more common to be used (Wang et al., 2007). After having found out mistakes, the receiver sends the data message containing serial number of false video frames and transmits correct frames instead of all frames.

ARQ method can reduce quantity of information and consummation of resource (Moore et al., 2004). Although ARQ is an efficient way, it isn't fit to the situation of narrow bandwidth. When channel is obstructed, it's easy to lose packets and frames and consequently increase the quantity of rollback data packets; the condition of network becomes worse and worse. Besides, since the rate of packet-loss increases, the receiver has to send back repeatedly the same data, and it will cause serious time-delay. Finally, ARQ can not assure the real-time transportation.

\subsection{Forward Error Correction}

Forward Error Correction (FEC) method uses simplex communicating channel style, the receiver operates the total tasks of checking error and restoring video (Wang et al., 2007). In the process, the sender creates error correct codes to support the receiver to rectify mistakes, such as RS code or $\mathrm{BCH}$ code. In the process of checking, the receiver doesn't send reports of checking result and the sender keep sending streams all the time.

Advantages of FEC are that, because receiver checks mistakes by itself and sender takes no operations to adjust the transmitting rate and send correct data, it will reduce the times of communication between double sides and decrease the redundant data to enhance utilization rate; Even though some redundant codes lose in the transportation, it effects little to the quality of video. 
Disadvantages of FEC are that, firstly, if the bandwidth changes suddenly, the sender can't percept the change of channel and keeps the stable speed to transmit, which leads to lose many packets (Liu et al., 2004); secondly, it will affect terribly checking error. And because it doesn't send correct data, video will be hard to restore (Gu et al., 2002). thirdly, error correct codes contain lots of information of motion compensation, there will be bad obstruction as large volume of correct codes.

\section{DESIGN OF ADAPTIVE ERROR-CORRECTION SOLUTION}

\subsection{Error-Retransmit solution based on RDP protocol}

In order to ensure complete transmission in the unstable circumstance, the receiver needs to check error for video data, as soon as finding out mistake, the receiver should send report containing the result of rectification to request the sender to send the correct data back. It needs the support of transmitting protocol to achieve feeding back.

It's known that, traditional ARQ method designed on the basis of TCP, which sets up mechanisms that provide safe-guarantee to the peer-to-peer transportation ( Lin et al., 2002). But because TCP uses complex process of interaction and tedious retransmitting mechanism leading to heavy burden for resource and serious time-delay, so TCP is not available to the real-time transportation of huge video streams. With the development of web technologies, transmitting protocols have been improved greatly. Nowadays, there have been many protocols that are suitable to support the transportation of mass streams, and RDP (Reliable data protocol) gets the best comprehensive effect (Hu et al., 2004).

RDP provides each transmitting layer with duplex communicating channels, it tries to reliably send all user information and show mistakes if there are some failures in the process. Its extended IP data service ensure reliable transmission, and it takes advantages of serial number and correcting code packaged in the head of data packet to detect and remove the false data. Compare to TCP, RDP is convenient to user, because it supply simple functions for controlling and buffering and managing (Ma et al., 2007). For the transportation of stream, RDP provide powerful index-searching function containing synchronization code and serial number to load frame timing and spacing order. It is available for the receiver to finish the sorting and complex statistic of losing frames. The structure of RDP packet is shown in Fig.1. In view of perfect functions, this paper sets up SARQ method on the base that uses RDP data packets to record the list of video streams. 


\begin{tabular}{|c|c|c|c|c|c|c|c|}
\hline SWN & $\mathrm{ACK}$ & $\mathrm{ESK}$ & RST & NILL & 0 & Ver No & Header Length \\
\hline \multicolumn{8}{|c|}{ SourcePort } \\
\hline \multicolumn{8}{|c|}{ DestinationPort } \\
\hline \multicolumn{8}{|c|}{ Data Length } \\
\hline \multicolumn{8}{|c|}{ Sequence Number } \\
\hline \multicolumn{8}{|c|}{ Acknowledgement Number } \\
\hline \multicolumn{8}{|c|}{ Checksum } \\
\hline \multicolumn{8}{|c|}{ Variable header area } \\
\hline
\end{tabular}

Fig.1: The structure of RDP packet

Video transportation is commonly divided into two styles: video-frame model and video-packet model, they use individually particular ways to transmit streams ( $\mathrm{Xu}$ et al., 2005). In order to be compatible with two modes, this paper improves original formation of RDP data packets deleting all useless features and adding some user-defined characters. In case that one packet may encapsulate several video streams, the receiver has to verify that all packets have been gotten completely. So, this paper proposes adding data sequence feature to RDP data packets, which is used to record list of video frames. As removing some useless data items, the volume of packets become a bit small so that it can avoid obstructing the channel because of large volume. Improved structure of RDP data packet is shown in Fig.2.

\begin{tabular}{|c|c|c|c|}
\hline SYN & ACK & EAK & RST \\
\hline \multicolumn{3}{|c|}{ SourcePort } \\
\hline \multicolumn{3}{|c|}{ DestinationPort } \\
\hline \multicolumn{3}{|c|}{ Data Length } \\
\hline \multicolumn{3}{|c|}{ Data Sequence } \\
\hline \multicolumn{3}{|c|}{ Checksum }
\end{tabular}

Fig. 2: Improved structure of RDP data packet

As sending video frames and packets, the receiver will add with RDP data packet and record detailed information. After getting video packets, the receiver firstly examines additional information in RDP data packet, and check error. If the object transmitted is real-time stream, the receiver tries to find whether streams are in the right order or lost by the SYN and ACK item. Taking advantage of data sequence, it is clear whether stream frames encapsulated in video packet were lost. Finishing checking error, the receiver will record the list of serial number of frames or packets lost, and send feed-back report to inform the sender sends the correct data again.

For instance, in order to adjust the volume of data packets, the receiver will package one key frame and several referenced frames in one packet, as 
shown in Fig.3, data sequence item will record their serial number: 1-0 and 1-1 and so on. Having attained packets, the receiver will examine whether the current order of frames is matching the original order, if some frames were lost, the receiver will request the sender to transmit data again according to the data sequence.

$$
\begin{array}{|c|c|c|c|c|}
\hline \mathrm{I} & \mathrm{p} & \mathrm{p} & \mathrm{p} & \mathrm{p} \\
\hline 1-0 & \# 1-1 & \# 1-2 & \# 1-3 & \# 1-4 \\
\hline
\end{array}
$$

Fig.3: The structure of encapsulated frame packet

\subsection{The solution of cutting apart dynamically image based on varying code-rate}

Traditional FEC method is a way that with the constant speed, the receiver takes charge of checking error and correcting image by itself (Mei et al., 2004). This method lacks elemental communication between two sides that are responsible to the transportation, even though there are critical mistakes, which are hard to rectify, the receiver is difficult to modify the transmitting speed, because it can't get any report of the situation of network (Lin et al., 2007). The final result is that there are more and more false streams and the transportation is tough to continue. So, this paper proposes a new solution improved FEC based on predicting current sending rate. The receiver will take advantage of RDP protocol to send periodically RDP feed-back reports, which are used to trace the changing trend of channel. In accordance to reports, the receiver calculates the rational speed to ensue the normal transmission. This paper uses TFRC (TCP-Friendly Rate Control) algorithm to evaluate ideal speed of transmission. The formula of TFRC is depicted as followed.

$$
T=\frac{S}{R T T \sqrt{\frac{2 b P}{3}}+t_{-} R T O \sqrt{\frac{3 b P}{8}}\left(1+32 P^{2}\right)}
$$

$T$ is upper limit of sending speed, $R T T$ is round trip time, $t_{-} R T O$ is time of overtime retransferring, $P$ is pocket loss event ratio, $S$ is size of data packet. If the receiver can get data in a $R T T$ interval, it will feed back to the sender with packet-loss rate, and ensue that the sender can obtain continuingly this parameter.

Traditional FEC uses the motion estimation of all pixels of an image and formats RS correcting code containing estimation of the time and space region. In the process of checking error, the receiver examines whether the motion of pixels accord with original estimation, when there are some false motion, it can be corrected by the RS code (Gao et al., 2006). This 
approach is more available to use in the situation that the bandwidth is quite good, but, because high redundancy of RS code will heavy the transferring burden of channel, if the bandwidth becomes very narrow, images will not be reduced resulting from loss of lots of RS code. Moreover, FEC will increase complexity of reducing image, particularly as the receiver could not get whole RS code. So, in the premise of speed estimation, this paper proposes a checking solution by dynamical image segmentation: SFEC.

In order to reduce the influence of high redundancy of correcting code, SFEC expends the region of pixels and uses the estimation of image blocks instead of pixels. Through dynamically cut apart the image, it will decrease the complexity of RS code. This paper adopts even-square dynamic algorithm to divide an image into several fragments, such as $2 \times 2 、 4 \times 4 、 8$ $\times 8$ and so on.Fig. 4 shows segments of the image.

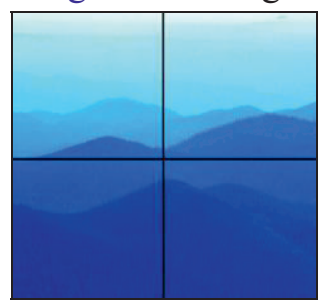

(a) $2 \times 2$ format

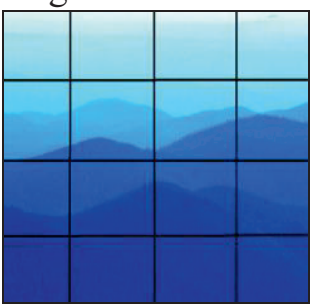

(b) $4 \times 4$ format

Fig. 4: Image segments operated by SFEC

The first step is, Calculate the expected transmitting speed. At first set $k$ regions of changing blocks, and through several experiments measure the average value of each changing block, and set the weight according to the frequency of appearance of each region, finally calculate the theoretic speed value.

The second step is, during the transmission, firstly estimate value of $p_{t}$ that stands for current transmitting speed, and calculates the segmenting coefficient depicted with $h$.

$$
h=\frac{\sum_{i=1}^{k} m_{i} p_{c}}{p_{t}}
$$

Where:

$$
0<m_{i}<1, \sum_{i=1}^{k} m_{i}=1
$$

The third step is, If $\mathrm{h}<=1$, it certificates the current speed is more than expected speed, and this situation is fit for real-time transmission, it is unnecessary to slit the image; If $\mathrm{h}>1$, it stands for that the current communicating channel is terrible, and $\mathrm{h}$ is larger as packet-loss rate is increasing, the possibility of losing correcting code is larger.

Calculate the quantity of video blocks: 


$$
N=\left(\frac{1}{l_{a} \times h}\right)^{2}
$$

Where:

$$
0<l a<1,1 /(l a \times h) \text { is even }
$$

$l_{a}$ is relative coefficient of segments, which is related to the size and the style of image.

$N$ is the quantity of blocks, inverse with the $h$ and is proportional with $p_{t}$, it stands that the quantity of blocks becomes less as the current speed is lower, and decreases redundancy of RS by extending the region of pixels and reducing the resolution of image.

\subsection{Realization of adaptive solution}

This paper proposes an adaptive error-check solution based on estimating the change of packet loss rate. Before starting transmitting, it should set a rational threshold value accepted by both the receiver and the sender, considering the request of the quality of video and the real-time transportation. During the transmission, supported by RDP protocol to create the network connection, the sender captures the feed-back data packet containing the list of video packets obtained by the receiver at last transmission, and uses formula 3.4 to calculate the current packet-loss rate and transmitting speed by TFRC. Finally, according to packet-loss rate, adjust the transmitting approach.

Calculate the packet-loss rate.

$$
r_{\text {loss }}=\frac{n_{t}-n_{r}}{n_{t}} \times 100 \%
$$

$r_{\text {loss }}$ is packet-loss rate, $n_{t}$ is the number of total packets transmitted, $n_{r}$ is number of all packets received.

If packet-loss rate is less than threshold value, it's better to use SARQ. The sender calculates the sending speed at first and the sender checks error and rectifies mistake. Finishing upper work, the sender sends video frames and correcting codes that have been operated by the algorithm of imagesegment with the predicting speed. The receiver takes responsibility of checking error and reduce image, and send the feed-back report to the sender. The detailed process is shown as Fig.5. 


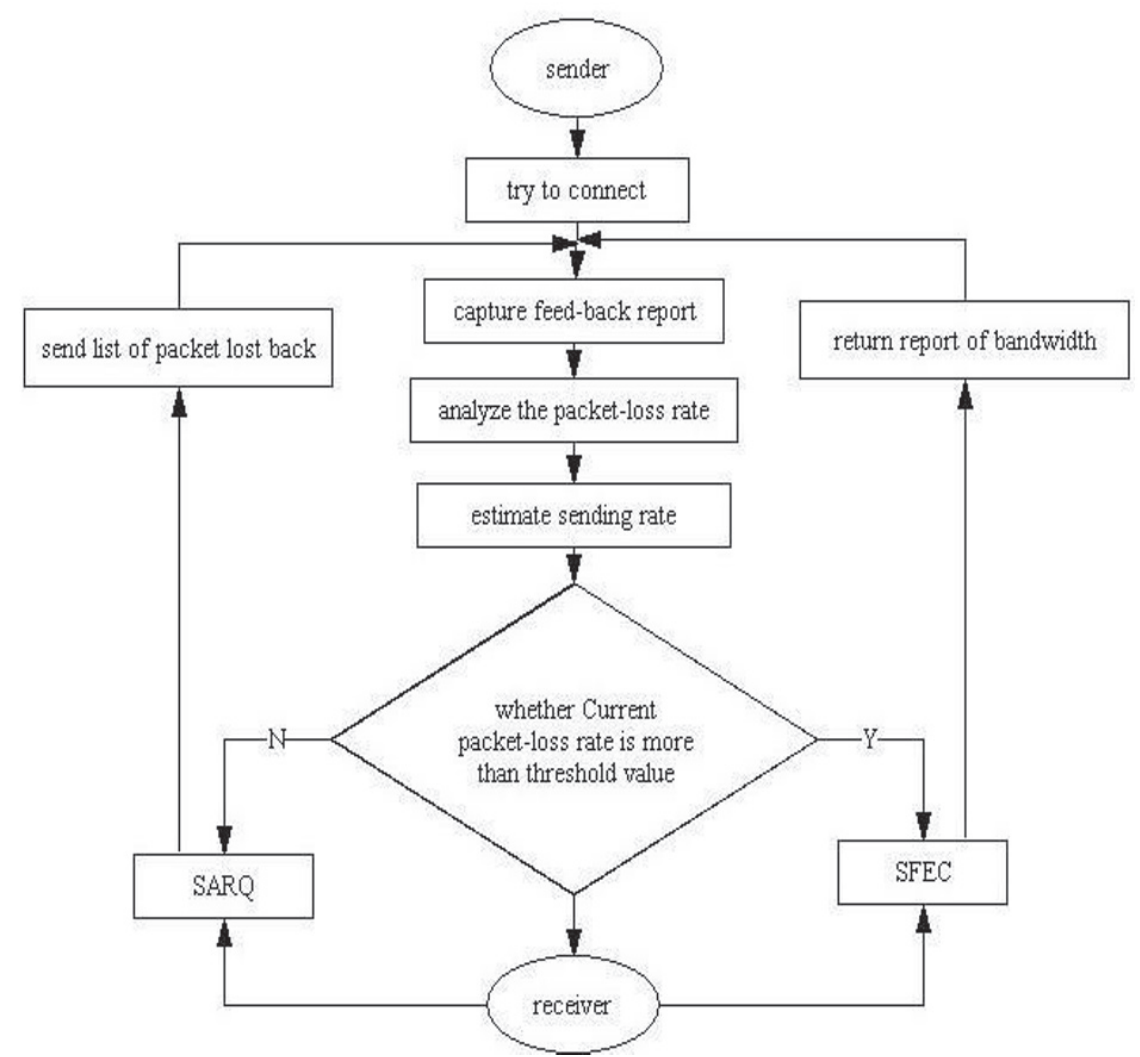

Fig. 5: Process of the realization of adaptive solution

\section{THE SIMULATION AND ANALYSIS}

This paper does tests with video segments of "Foreman", whose format is QCIF and the style of YUV is 4:2:0 and the coding frame number is 20 and post-code frame frequency is 10FPS. The switching time is subjected to exponential distributions, and the value is $400 \mathrm{~ms}$. The original speed is set by $512 \mathrm{kbps}$. The threshold value of packet-loss rate is 0.1 and this value is controlled in the region from 0 percent to 30 percent. The result is shown as Table1. and Table2. and Fig.6.

Table 1.The comparison of the result of two methods as the packet-loss rate keeps from 0 to $10 \%$.

\begin{tabular}{ccc}
\hline & SARQ & ARQ \\
\hline PSNR $(\mathrm{dB})$ & 37.14 & 36.88 \\
Bit rate $(\mathrm{Kbps})$ & 29.45 & 25.13 \\
Coding time $(\mathrm{s})$ & 37.92 & 45.17 \\
\hline
\end{tabular}


Table 2.The comparison of the result of two methods as the packet-loss rate keeps from $10 \%$ to $30 \%$.

\begin{tabular}{ccc}
\hline & SFEC & FEC \\
\hline PSNR $(\mathrm{dB})$ & 35.11 & 28.46 \\
Bit rate $(\mathrm{Kbps})$ & 26.29 & 20.36 \\
Coding time $(\mathrm{s})$ & 43.73 & 54.82 \\
\hline
\end{tabular}

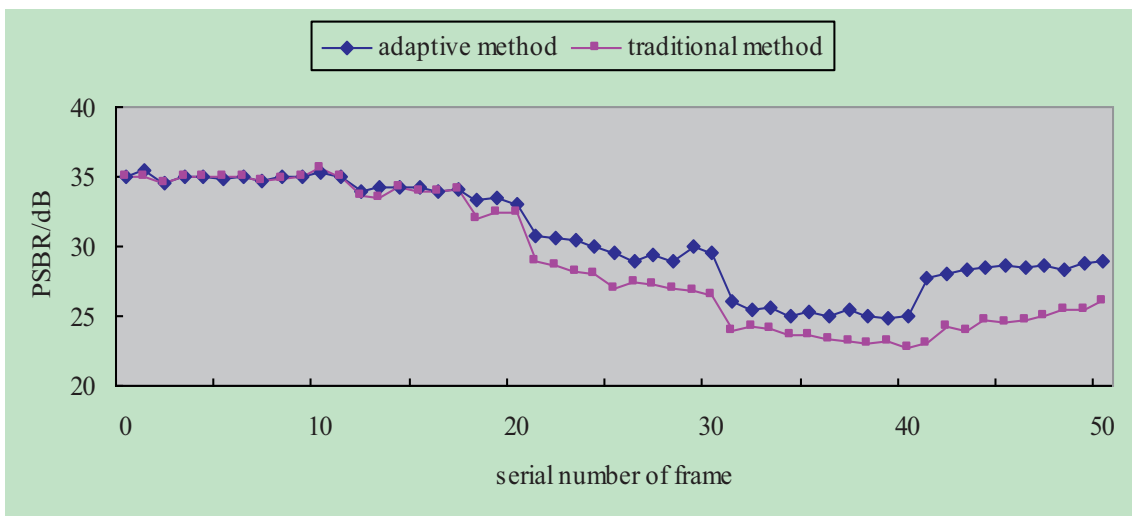

Fig. 6: Comparison of signal-to-noise ratio as the packet-loss rate keeps from $10 \%$ to $30 \%$

Under the condition that packet-loss rate keeps from 0 to 10 percent, we test with SARQ method. The receiver sends lists of false video streams with the improved format of RDP data packet. Compare to the traditional method, the result of experiment is not obvious, because the packet-loss rate is a bit low and the quality of channel is relatively good, but we also find the effect of SARQ is better. If the packet-loss rate is from 10 percent to 30 percent, we use SFEC to realize transportation and checking error. After estimating the trend of changing packet-loss rate, we slit image with two formats, such as $8 \times 8$ and $16 \times 16$. Compared with traditional FEC, the result certificates, not only at the aspect of optimization of sending speed but video quality, the adaptive solution can get better effort in the varying circumstance.

\section{CONCLUSION}

Unstable bandwidth is a main reason that affects the real-time transportation and the quality of video images. In the condition of low packet-loss rate using SARQ and the condition of high packet-loss rate using SFEC, to solve the problem, this paper proposes an adaptive error-check solution. In practice, this method can bring very good effects to the real-time transmission and the quality of video. 


\section{ACKNOWLEDGEMENTS}

This work is supported under the National Natural Science Foundation of China No. 60603027, the Soft Science Research Program of CSTC N0.2007CE9006 and the Bulk Warehouse Grain Quantity Recognition, a project (2007) funded by the Finance Bureau of Chongqing Municipality, P.R. China.

\section{REFERENCES}

B Moore, D Durham. J Strassner et a1. Information Model for Describing Network Device QoS Datapath Mechanisms. RFC 3670, 2004-01

Fan Xinnan. Xing Chao. Study of End to End Video Transmission Based 0n Internet, Computer Engineering, 2007, 33(3):218-220

Gao Peng, Chen Yongen. Research on Error Correct and Adaptive Error Concealment Algorithm for MPEG-2 in Video Communication, Computer Science, 2006,33(3):8486,204

Gu Jian, Yu Shengsheng, Zhou Jingli. Survey of Error Concealment Technique for Real-Time Video, Computer Engineering and Applications, 2002,38(10):57-59,118

Hu Weijun, Li Kefei. Error Resilient Video Coding for Video Streams Transmission, CHINA CABLE VISION, 2004, 2:25-28

Lin Ying, Ge Liang. Study on Real-time Video Transportation for National Grain Depot, CCTA 2007: 533-541

Liu Jieping, Yu Yinglin. A Scheme for Video Transmission Based on Multiple Description, Computer Engineering and Applications, 2004, 40(35):119-121

Ma Xin, YANG Xiaokang, Song Li. An Adaptive Temporal Error Concealment Technique, Journal of Image and Graphic, 2007, 12(10):1782-1784

Mei zheng, Li Jintao, An Adaptive Forward Error Correction Algorithm for Streaming Video, Journal of Software, 2004,15(9):1405-1412

Q Zhang, W W Zhu, Y Q Zhang. Channel-Adaptive Resource Allocation for Scalable Video Transmission Over 3G Wireless Network[J]. IEEE Transactions on Circuits and Systems for Video Technology.2004,14(8): 104 9-1063

Shan Y, Kalyanaraman S. Hybrid Video Downloading / Streaming over Peer-to-Peer Networks, Proc. of International Conference on Multimedia and Expo, 2003: 665-668

Wang Feng, Zhu Guangxi, Zhang Zhenming. Multi-product Code for FGS Video Transmission over IP-Based Wireless Networks, Computer Engineering and Applications, 2006, 29:143-146

Wang Ke, Liu Zhiqin. Strategy of Stream Media High Quality Transmission Based on P2P Overlay Network, Computer Engineering, 2007, 33(15):137-139

$\mathrm{Xu}$ Fan, Zeng Zhiyuan,MPEG-2 Internet Video Using TCP-friendly Rate Control and Forward Error Correction, Microcomputer Applications, 2005, 26(5): 556-559

Zhang Qian, Zhu Wenwu, Zhang Yaqin. Resource Allocation for Multimedia Streaming over the Internet Multimedia, I EEE Transactions, 2001, 3(3): 339-355 